\title{
Mechanism of Population Dynamics
}

\author{
Ron W Nielsen \\ PO Box 178, Bond University, Qld, 4229, Australia. \\ Email: nielsens@iprimus.com.au
}

\begin{abstract}
Explicit assumption that the use of natural resources promoting and sustaining growth is proportional to the number of people living on the planet at any given time leads to a model, which reproduces the population data remarkably well.
\end{abstract}

We start with the familiar expression for the incremental increase (or decrease),

$$
d N(t)=f(t) N(t) d t
$$

where $f(t)$ is often assumed constant but generally it can be a function of time depending on growth-influencing factors.

If $f(t)=k$, where $k$ is a constant, the eqn (1) has the well-known solution

$$
N(t)=C e^{k t}
$$

where $C$ is related to the constant of integration. When $k>0$, the eqn (2) describes the exponential growth, such as the growth associated with the fixed 
compound interest or the economic growth with a fixed percentage. When $k<0$, it describes an exponential decrease, such as the radioactive decay or the removal of polluting gases from the atmosphere.

Malthus postulated that the growth of population depends on the availability of food (Malthus 1798). This dependence has to be incorporated in the expression for the incremental increase, which can be done by using an appropriate expression for $f(t)$. The interpretation of the eqn (1) is then that the incremental change depends not only on the number of elements in the sample, as for instance in the radioactive decay, but also on the growth-affecting agents.

We shall now make an explicit assumption that the use of natural resources promoting and sustaining growth is proportional to the number of people $N(t)$ living at any given time on the planet. The simplest dependence on $N(t)$ is the function directly proportional to $N(t)$, i.e. $f(t)=k N(t)$ where $k$ is a constant representing the average per capita consumption. However, to allow for some flexibility, constant $k$ can be replaced by a time-dependent form factor $g(t)$, i.e. by assuming that the per capita consumption of natural resources sustaining growth may depend on time. The function $f(t)$ takes then the following form:

$$
f(t) \equiv g(t) N(t)
$$


In principle, the function $g(t)$, which can be determined by fitting the data, can have any form. Strong variations of this function would indicate strong per-capita variation in the consumption of sustaining resources. In general, and particularly on the global scale, such strong variations should not be present. Thus, for the well-working model, the function $g(t)$ should to be either constant or it should be a slowly varying function of time.

The eqn (1) can now be rewritten as:

$$
\frac{d N(t)}{d t}=g(t)[N(t)]^{2}
$$

This is a simple differential equation, which has the following solution:

$$
N(t)=\frac{1}{-\int g(t) d t}
$$

The simplest solution is when $g(t)=k$, i.e. when $f(t)=k N(t)$ and is given by the first-order hyperbolic function:

$$
N(t)=\frac{1}{C-k t}
$$

with $C$ being a constant of integration. 
Fig. 1 compares the average values of the latest and the best set of the world population data (Manning n.d.) with the derived model. This compilation agrees well with the previous compilation (USBS n.d.) but it contains also some new sources. The most consistent set of data is claimed to be from the year 1650 (Caldwell and Schindlmayr 2002).

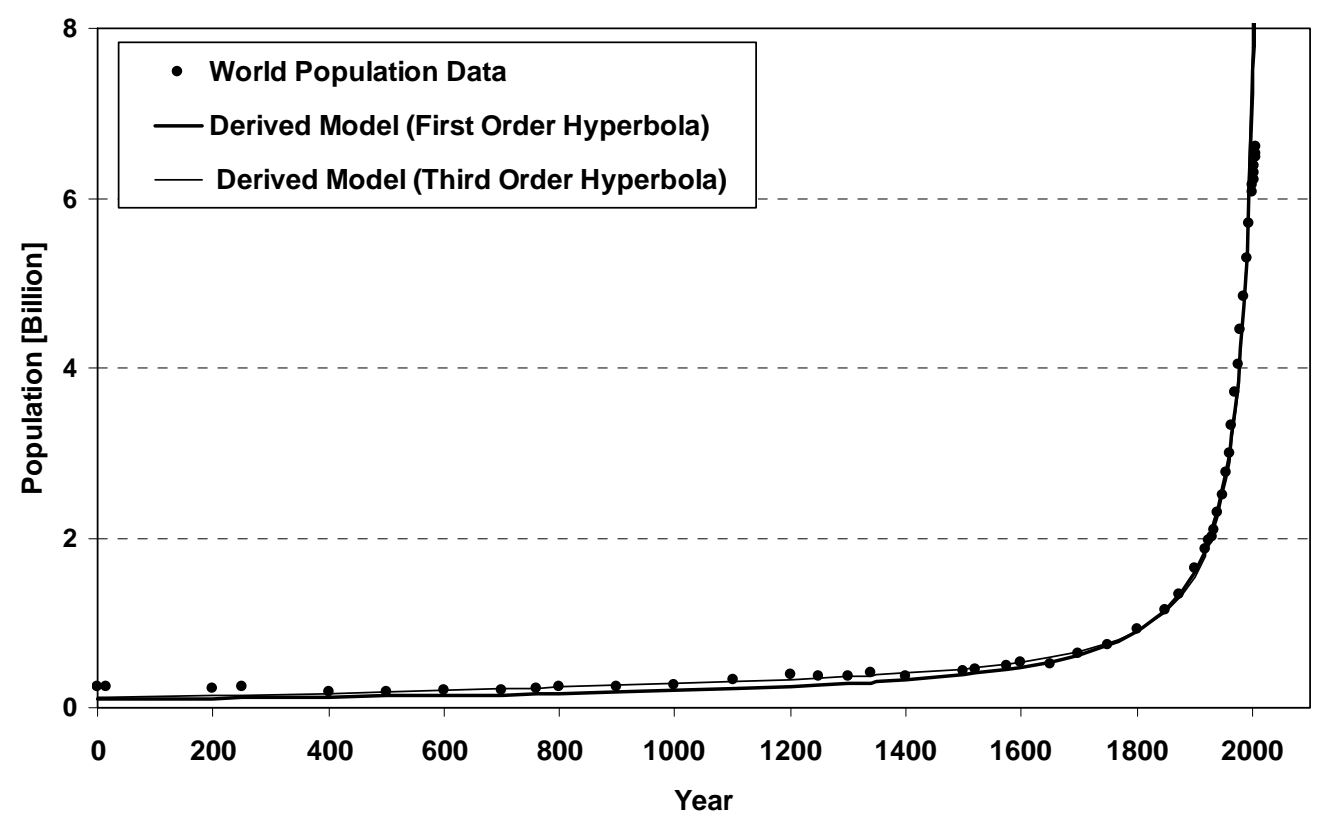

Fig. 1. The world population data (Manning n.d.) fitted using the derived hyperbolic model.

As can be seen, the derived model, which is based on a single and explicit mechanism, reproduces the world population data remarkably well. However, there is a small but clear discrepancy for the data below AD 400 and for the most recent data. It should be noted that the model has been also tested successfully using regional data (Maddison 2001). 
The fit to the world population data, generated by the first-order hyperbolic function (i.e. with $g(t)=$ constant) can be slightly improved by using a third-order hyperbola corresponding to $g(t)=a t^{2}+b t+c$. The coefficients for the best fit shown in Fig. 1 are: $a=4.2 \times 10^{-9}, b=-1.02 \times 10^{-5}$ and $c=0.0086$, which means that in this case also $g(t) \approx$ constant. As can be seen, the difference between the two fits is minimal. The average deviation of the data from the best hyperbolic fit is on average within the limits of $\pm 5 \%$.

The derived model and its excellent agreement with the data strongly suggest that that the growth of the world population has been controlled by a single dominating mechanism. Other factors may have had local influence but any temporary fluctuations caused by them were smoothed out by the prevailing driving force of using natural resources in proportion to the number of people living at any given time on the planet. This single mechanism has been dominating for at least the past 1500 years, i.e. between around $A D 400$ and the early 1900s. However, extended analysis, using the data down to $10,000 \mathrm{BC}$, indicates that this single mechanism has been the main driving force for the past 12,000 years.

The data displayed in Fig. 1 follow a characteristic but unfamiliar distribution, which is prone to misinterpretation. It is, however, just a hyperbolic distribution governed by a single and simple mechanism. The population has been increasing along this unfamiliar trajectory simply because the average per-capita 
consumption of natural resources sustaining growth was approximately constant. Some people consume more; some consume less. Per capita consumption might have varied in time, but its average value, as indicated by the data, remained approximately unchanged. Regional data (Maddison 2001) follow also hyperbolic distribution.

There appears to be no reason why this model, with its rather straightforward assumption, should not be applicable to other species. However, as any other model, it also has its limitations. It does not, for instance, account for the policycontrolled growth such as in China, or for a collective and voluntary decision to limit growth, as occurring in developed countries. Nevertheless, the data indicate that the natural tendency for the population dynamics is to be controlled by a single and simple mechanism, which generates hyperbolic growth.

\section{References}

Caldwell, J. C. and Schindlmayr, T. 2002. 'Historical Population Estimates: Unraveling the Consensus', Population and Development Review 28, 183.

Maddison, A. 2001, The World Economy: A Millennial Perspective, OECD, Paris. 
Malthus, T. R. 1798, An Essay on the Principle of Population as It Affects the Future Improvement of Society, with Remarks on the Speculations of Mr Godwin, M. Condorcet, and Other Writers, J. Johnson, London.

Manning, S Year-by-Year World Population Estimates: 10,000 B.C. to 2007 A.D., 2008, http://www.digitalsurvivors.com/archives/worldpopulation.php, accessed 5 April 2008.

USBS, Historical Estimates of World Populations, US Bureau of Statistics, 2008, http://www.census.gov/ipc/www/worldhis.html, accessed 5 April 2008. 


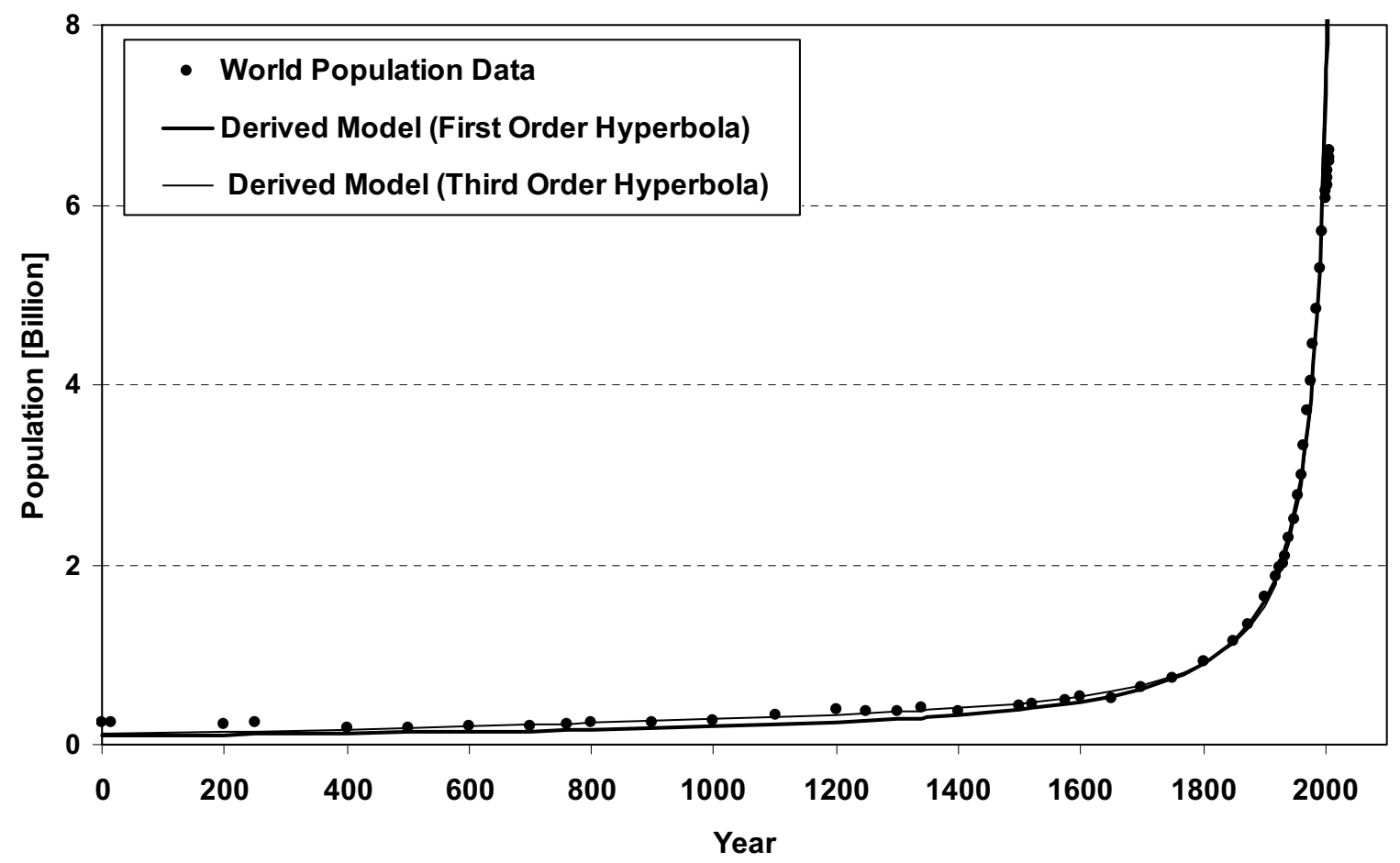

R W Nielsen, Population Dynamics, Figure 1 